\title{
Dynamics of liquid nanothreads: Fluctuation-driven instability and rupture
}

\author{
Chengxi Zhao $\odot$ and Duncan A. Lockerby ${ }^{*}{ }^{*}$ \\ School of Engineering, University of Warwick, Coventry CV4 7AL, United Kingdom \\ James E. Sprittles $\oplus^{\dagger}$ \\ Mathematics Institute, University of Warwick, Coventry CV4 7AL, United Kingdom
}

(Received 25 November 2019; accepted 19 February 2020; published 2 April 2020)

\begin{abstract}
The instability and rupture of nanoscale liquid threads is shown to strongly depend on thermal fluctuations. These fluctuations are naturally occurring within molecular dynamics (MD) simulations and can be incorporated via fluctuating hydrodynamics into a stochastic lubrication equation (SLE). A simple and robust numerical scheme is developed for the SLE that is validated against MD for both the initial (linear) instability and the nonlinear rupture process. Particular attention is paid to the rupture process and its statistics, where the "double-cone" profile reported by Moseler and Landmann [Science 289, 1165 (2000)] is observed, as well as other distinct profile forms depending on the flow conditions. Comparison to the Eggers' similarity solution [Phys. Rev. Lett. 89, 084502 (2002)], a power law of the minimum thread radius against time to rupture, shows agreement only at low surface tension; indicating that surface tension cannot generally be neglected when considering rupture dynamics.
\end{abstract}

DOI: 10.1103/PhysRevFluids.5.044201

\section{INTRODUCTION}

The interface dynamics of liquid threads is important to a range of technologies, such as inkjet printing [1], fiber manufacture [2], and drug delivery [3]. In classical fluid-dynamics theory there are two stages to liquid thread breakup where good analytic progress has been made [4]: (i) the initial (linear) instability generation and (ii) the nonlinear rupture dynamics.

The linear instability of a liquid cylinder is described by the classical results of Rayleigh and Plateau (referred to hereon as the RP theoretical framework): Plateau [5] predicted a critical wavelength, $\lambda_{\text {crit }}=2 \pi r_{0}$, beneath which all interface disturbances decay; and Rayleigh [6] found the fastest growing mode above this threshold, e.g., for the inviscid case, $\lambda_{\max }=9.01 r_{0}$ (or wave number $\left.k_{\max }=0.697 / r_{0}\right)$.

For the stage leading to rupture, different scaling theories [7] have been developed to predict the dynamics in three regimes: the inertial regime [8,9], where inertial and capillary forces are comparable (viscous forces are weak); the viscous regime [10,11], where viscous and capillary forces are comparable (inertial forces are weak); and the viscous-inertial universal regime, where viscous, capillary, and inertial forces are all comparable [12]. These classical theories are supported

\footnotetext{
*D.Lockerby@Warwick.ac.uk
}

J.E.Sprittles@Warwick.ac.uk

Published by the American Physical Society under the terms of the Creative Commons Attribution 4.0 International license. Further distribution of this work must maintain attribution to the author(s) and the published article's title, journal citation, and DOI. 
by numerous macroscopic experiments [4] and there is recent evidence that the transitions between these regimes is rather complex [7,13,14].

Motivated by the emergence of micro/nanofluidic technology (e.g., lab-on-a-chip devices [15], $3 \mathrm{D}$ printing [16], and nanodevice fabrication [17]), the validity of these classical theories at the microscale and nanoscale has recently been brought into question. In a pioneering article, Moseler and Landman [18] used molecular dynamics (MD) simulations of nanoscale jets to discover a rupture profile not described by macroscopic theory: A "double-cone profile" was observed at rupture, a phenomenon attributed to thermal fluctuations that are negligible at usual engineering scales. The importance of thermal fluctuations at these scales has been confirmed by physical experiments using specially prepared low-surface-tension liquid-liquid combinations that enhance fluctuations $[19,20]$ and further MD simulations $[21,22]$.

The double-cone profile was also predicted by solutions to stochastic lubrication equations (SLE) [18]. The SLE are derived by applying a lubrication approximation to the Landau-Lifshitz Navier-Stokes equations (LLNS), which are the Navier-Stokes equations plus stochastic terms used to model the thermal fluctuations [23]. The SLE have two very significant advantages over MD: (i) they are far less computationally intensive to solve numerically and (ii) they are amenable to theoretical analysis that provide more insight into the process. In relation to the latter, in Zhao et al. [24], a framework was developed for modeling (linear) interface instability in the presence of thermal fluctuations (named the SLE-RP). At the nanoscale, the SLE-RP shows that Plateau stability can be violated and $k_{\max }$ predicted by classical theories is significantly modified (notably becoming time dependent). Interestingly, for rupture dynamics, Eggers [25] has derived a "nanoscale" similarity solution that incorporates thermal fluctuations and is able to reproduce the double-cone profile observed by Moseler and Landman [18]. However, the SLE has never been focused on this case to study the accuracy of this solution and the validity of the assumptions made (e.g., negligible surface tension).

Numerical solutions to the SLE offer much broader applicability than the analytic results described above, and can be obtained at a small fraction of the computational cost of MD. A set of closely related stochastic equations are well studied for thin-film flows [26-30] and thermally activated vapor-bubble nucleation [31]. But, surprisingly, there are no detailed numerical SLE studies in the literature for liquid thread rupture. In previous work (e.g., Refs. [18,22]) only qualitative comparisons between MD and selected SLE realizations were presented. However, the SLE are stochastic, and many independent solutions are needed to (i) understand the statistics of the rupture process, and (ii) verify that these statistics are well described by the SLE (in comparison to MD). Filling this gap in knowledge is the primary contribution of the work presented here.

The article is laid out as follows. In Sec. II the SLE are introduced (Sec. II B), a simple, yet robust, scheme for their numerical solution is proposed (Sec. IIC), and its convergence characteristics demonstrated (Sec. II D). In Sec. III, numerical SLE solutions are verified against known analytical results and validated against MD calculations (introduced in Sec. II A); first for initial (linear) instability growth (Sec. III A), and second for nonlinear growth of disturbances to the point of rupture (Sec. III B). In Sec. IV we use the SLE solver to (i) provide a deeper understanding of the impact of fluctuations on rupture dynamics and (ii) reach cases that will be computationally intractable for MD.

\section{MODELS AND NUMERICS}

In this section we introduce the stochastic lubrication equations and present a numerical method for their solution. Given the stochastic and nonlinear nature of the equations, we dedicate some time to demonstrating the scheme's robustness to increasing numerical resolution. First, though, we give details of the molecular dynamics simulations presented in this paper, which are used in Sec. III for validation of the numerical SLE solutions. 


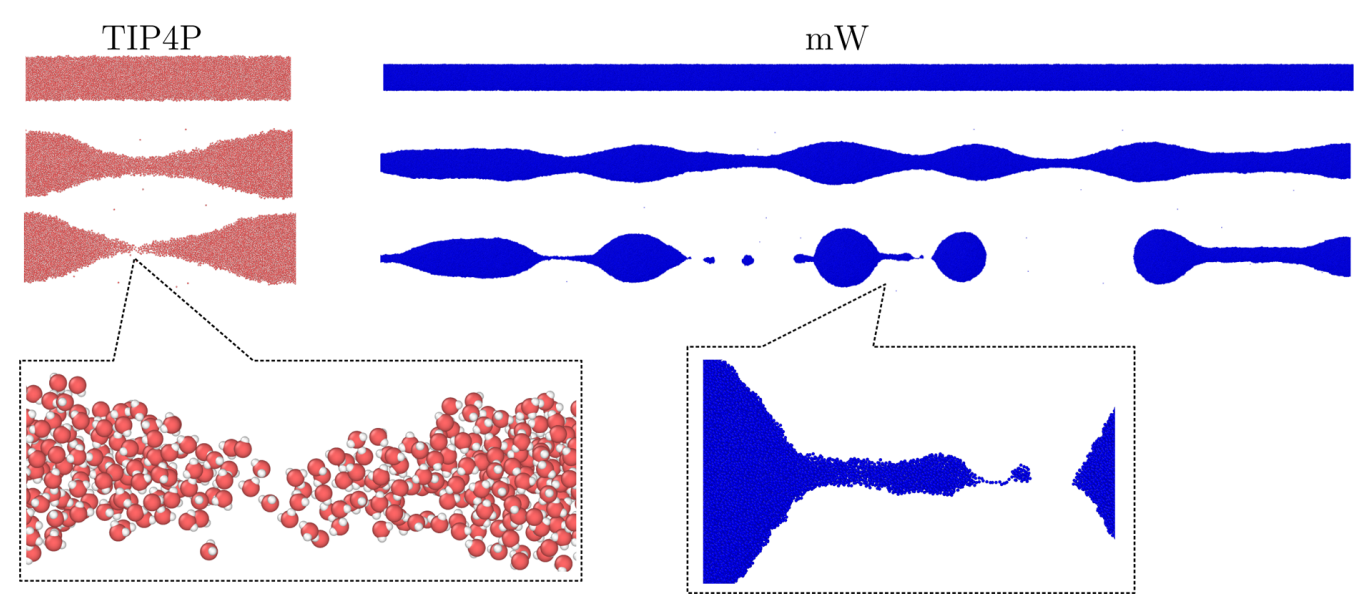

FIG. 1. MD simulations using the different molecular models for water. Left panel: rupture dynamics of a short thread predicted by the TIP4P/2005; right panel: perturbation instabilities of a long thread modelled by the coarse grained $\mathrm{mW}$ model. The details of these models are introduced in Appendix A.

\section{A. Molecular dynamics}

The MD simulations of this work are performed in LAMMPS [32] on nanoscale threads of water. The simulation box extends $10 r_{0}, 10 r_{0}$, and $L$ in the $x, y$ and $z$ directions, respectively, where $r_{0}$ is the initial thread radius and $L$ is the thread length. The liquid thread is placed in the center of the domain, and there are periodic boundary conditions imposed in all three directions.

The initial configuration is cut from a liquid bulk, created from an equilibrium NVT simulation with a Nosé-Hoover thermostat set at a specific temperature. The same ensemble and thermostat is used for the main simulations.

The Green Kubo method $[33,34]$ is applied to calculate the dynamic viscosity by integration of the time-autocorrelation function of the off-diagonal elements of the pressure tensor $P_{i j}$ so that

$$
\mu=\frac{V_{\text {bulk }}}{k_{B} T} \int_{0}^{\infty}\left\langle P_{i j}(t) \cdot P_{i j}(0)\right\rangle d t \quad(i \neq j),
$$

where $V_{\text {bulk }}$ is the volume of the bulk fluid, $k_{\mathrm{B}}$ is the Boltzmann constant, and $T$ is temperature. The pressure tensor components are obtained using the definition of Ref. [35] and the angular brackets indicate the expectation.

The surface tension is calculated from the profiles of the components of the pressure tensor in a simple liquid-vapour system, using the mechanical definition [36]:

$$
\gamma=\frac{1}{2} \int_{0}^{L_{z}}\left[P_{\mathrm{n}}(z)-P_{\mathrm{t}}(z)\right] d z,
$$

where $L_{z}$ is the length of the MD domain, and subscripts $\mathrm{n}$ and $\mathrm{t}$ denote normal and tangential components, respectively. These methods are well known and have been validated by comparing MD results $(\mu$ and $\gamma$ ) with experimental data over a range of temperatures (see Refs. [37,38]).

In the present work, liquid water is chosen because of its wide applications and its ability to create a large range of material properties [39] due to its stable liquid phase over a wide temperature range. The detailed properties (e.g., temperature, surface tension, viscosity) will be listed in the relevant sections. For the instability validation cases in Sec. III A (requiring long cylinders) a coarse-grained water molecule model, known as $\mathrm{mW}$ [40], is adopted (for computational efficiency); whereas for the breakup validation cases in Sec. III B, the TIP4P/2005 water model [41] is used (thus achieving a more accurate result). Selected MD realizations of both models are shown in Fig. 1. 


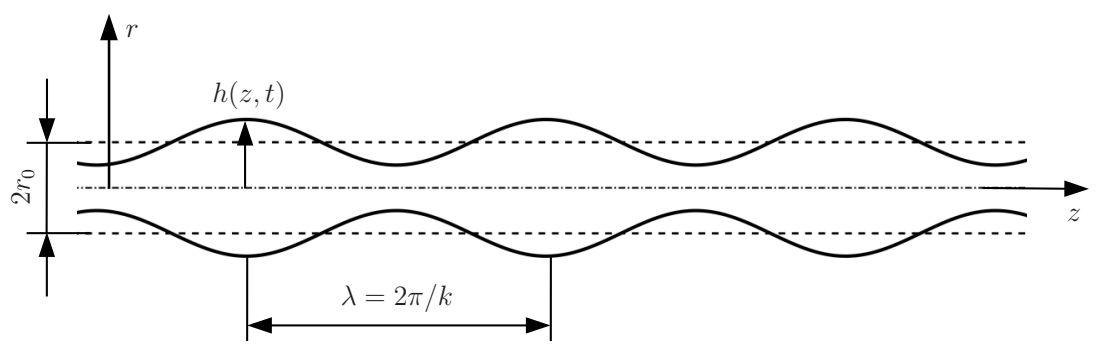

FIG. 2. Schematic of a liquid thread with a perturbed interface.

\section{B. Stochastic lubrication equations (SLE)}

The SLE were derived by Moseler and Landman [18], who applied a lubrication approximation to the axisymmetric LLNS (also known as the stochastic Navier-Stokes equations) allowing the dynamics of the interface to be described by the thread radius $h(z, t)$ and a velocity $u(z, t)$ (see Fig. 2). Modified stochastic lubrication equations, where a dependence of the evaporationcondensation flux on the presence of a surrounding gas is incorporated into the continuity equation, have yielded results agreeing well with MD [22]. However, in this work we restrict our attention to the orginal SLE.

To identify the governing dimensionless parameters, we use the following variables as scales of length, time, velocity and pressure, based on (but not confined to) a balance of inertial and surfacetension forces:

$$
h=\tilde{h} / r_{0}, \quad t=\tilde{t} / \sqrt{\rho r_{0}^{3} / \gamma}, \quad u=\tilde{u} / \sqrt{\gamma /\left(\rho r_{0}\right)}, \quad p=\tilde{p} /\left(\gamma / r_{0}\right)
$$

where $\tilde{h}, \tilde{t}, \tilde{u}$, and $\tilde{p}$ represent the dimensional interface height, time, velocity and pressure, respectively (note that the dimensional material parameters are not given tildes). The dimensionless SLE are written as follows:

$$
\begin{gathered}
\partial_{t} u=-u u^{\prime}-p^{\prime}+3 \mathrm{Oh} \frac{\left(h^{2} u^{\prime}\right)^{\prime}}{h^{2}}+\sqrt{\frac{6}{\pi}} \mathrm{Th} \sqrt{\mathrm{Oh}} \frac{(h \mathcal{N})^{\prime}}{h^{2}}, \\
\partial_{t} h=-u h^{\prime}-u^{\prime} h / 2,
\end{gathered}
$$

with the full Laplace pressure retained:

$$
p=h^{-1}\left(1+\left(h^{\prime}\right)^{2}\right)^{-\frac{1}{2}}-h^{\prime \prime}\left(1+\left(h^{\prime}\right)^{2}\right)^{-\frac{3}{2}}
$$

where primes denote differentiation with respect to $z$, and $\mathcal{N}$ is a standard Gaussian random variable - $\mathrm{a}$ model for thermal fluctuations. The nondimensional quantity $\mathrm{Oh}=\mu / \sqrt{\rho \gamma r_{0}}$ is the Ohnesorge number, which relates the viscous forces to inertial and surface-tension forces (where $\mu$ is the liquid dynamic viscosity, $\gamma$ is surface tension, and $\rho$ is liquid density). The Ohnesorge number is all that is needed to characterise the dynamics of free macroscopic threads, but here we obtain an additional dimensionless quantity: the thermal-fluctuation number, $\mathrm{Th}=l_{\mathrm{T}} / r_{0}$, to express the relative intensity of interface fluctuations, where $l_{\mathrm{T}}=\sqrt{k_{\mathrm{B}} T / \gamma}$ is the characteristic thermal fluctuation length and $r_{0}$ is the initial thread radius. When $\mathrm{Th}=0$, the classical model (LE) [42] is recovered.

One of the purposes of the simulations presented in Sec. IV is to explore the combined influence of Oh and Th on different aspects of thread dynamics. 

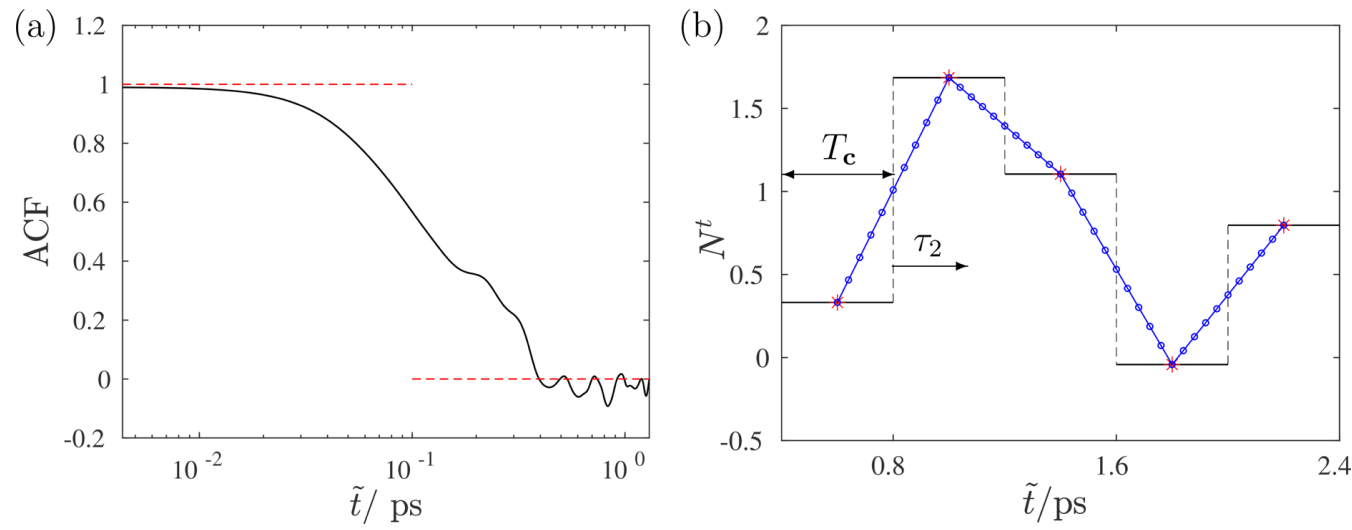

FIG. 3. (a) The auto-correlation function (ACF) for shear stress, obtained from an MD simulation of a periodic cube of liquid; (b) an illustration of the linear interpolation used in evaluating the temporal stochastic term $N^{t}$.

\section{Numerical scheme for the SLE}

By construction, after enforcing the fluctuation-dissipation balance, the covariance of the stochastic term in Eq. (5) is

$$
\left\langle\mathcal{N}(z, t) \mathcal{N}\left(z^{\prime}, t^{\prime}\right)\right\rangle=\delta\left(z-z^{\prime}\right) \delta\left(t-t^{\prime}\right),
$$

where the presence of a Dirac delta function ensures infinitely small temporal/spatial correlation functions (i.e., the noise term is temporally and spatially uncorrelated). To represent $\mathcal{N}$ numerically, we introduce computer-generated random numbers, $N_{i}^{t}$, that are normally distributed with zero mean and unit variance. The delta function in Eq. (7) can be approximated by a $2 \mathrm{D}$ rectangular (boxcar) function (in $t$ and $z$ ) that is nonzero over a time step $(\Delta t)$ and grid spacing $(\triangle z)$. The amplitude of the rectangular function, $1 /(\Delta t \Delta z)$, is such that the integral properties of the delta function are preserved, i.e., $\int_{-\infty}^{\infty} \int_{-\infty}^{\infty} \delta(z, t) \mathrm{d} z \mathrm{~d} t=1$ [43]. The complete noise term is thus discretized by

$$
\mathcal{N} \approx N_{i}^{t} / \sqrt{\triangle t \triangle z}
$$

Equation (8) provides robust and accurate numerical performance when used in conjunction with linear equations, e.g., one-dimensional LLNS [44] or for the linearised SLE. However, the full SLE are nonlinear (including the stochastic driving force: $(h \mathcal{N})^{\prime} / h^{2}$ ), which creates stability issues that exacerbate as $\Delta z$ and $\Delta t$ become smaller and the amplitude of noise becomes larger [see Eq. (8)]. Consequently, for some cases, it is impossible to achieve a spatially and temporally resolved result (i.e., one that converges as $\Delta z \rightarrow 0$ and $\Delta t \rightarrow 0$ ).

As a straightforward solution to this problem, we propose a numerical method where, beneath a certain scale, the noise becomes spatially and temporally correlated; thus remaining finite as $\triangle z \rightarrow 0$ and $\Delta t \rightarrow 0$. MD results show that this 'correlation scale' is much smaller than any scale of interest to the current paper, but we cannot be sure this will always be the case.

While this solution is largely pragmatic in nature, it actually reflects the physics better than uncorrelated noise. Figure 3(a) shows the temporal autocorrelation function of shear stress fluctuations in a bulk liquid, as calculated by MD (in a $3 \mathrm{~nm}^{3}$ periodic cube of TIP4P water at $T=340 \mathrm{~K}$ ). Notably, when timescales are much less than a picosecond the fluctuations become correlated; we find a similar situation in the spatial fluctuations of stress in MD.

Motivated by these MD results, we introduce a correlation timescale, $T_{\mathrm{c}}$, and correlation length scale, $L_{\mathrm{c}}$, into our SLE simulations. These correlation scales must be larger than the time step and grid spacing, respectively. Beneath the correlation scales, a simple linear interpolation between uncorrelated random noise at the end points of the correlation interval is applied (as illustrated 

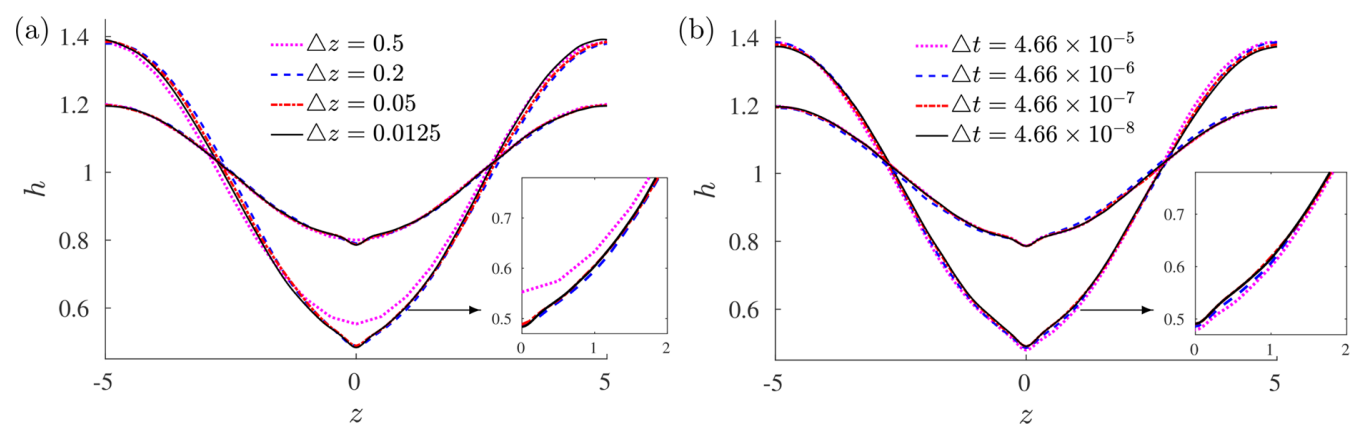

FIG. 4. Ensemble-averaged interface profiles at two time instances $\left(t_{1}=4.66 \times 10^{-2}\right.$ and $\left.t_{2}=1.69\right)$ for (a) decreasing grid-size with fixed time step $\left(\triangle t=4.66 \times 10^{-7}\right)$ and (b) decreasing time step with fixed grid size $(\triangle z=0.05)$.

in Fig. $3 \mathrm{~b}$ for temporal noise). The uncorrelated noise (which is interpolated between) has a mean of zero and a variance $1 /\left(T_{\mathrm{c}} L_{\mathrm{c}}\right)$. See Appendix B for a derivation.

Note, a spatially correlated, but temporally uncorrelated, noise model has been developed by Grün [26] for the stochastic thin-film equation. The approach we present above has the advantage of simplicity, compares well to the results of Grün's model and has the capability to produce spatiotemporally correlated noise as well.

To solve the full nonlinear SLE, we use the MacCormack method [45], a simple second-order explicit finite difference scheme in both time and space. The solution at each time level is defined by two arrays, $\left\{h_{i}\right\}_{i=1}^{n}$ and $\left\{u_{i}\right\}_{i=1}^{n}$. Here, $n$ is the number of mesh points. The time-derivative terms are approximated by $\left(h_{i}^{t+1}-h_{i}^{t}\right) / \Delta t$ and $\left(u_{i}^{t+1}-u_{i}^{t}\right) / \Delta t$. The numerical method proceeds in two steps: a predictor step,

$$
\left(\begin{array}{l}
\overline{u_{i}^{t+1}} \\
\overline{h_{i}^{t+1}}
\end{array}\right)=\left(\begin{array}{l}
u_{i}^{t} \\
h_{i}^{t}
\end{array}\right)+\mathbf{F}\left(u_{i}^{t}, h_{i}^{t}\right) \triangle t
$$

and a corrector step,

$$
\left(\begin{array}{l}
u_{i}^{t+1} \\
h_{i}^{t+1}
\end{array}\right)=\left(\begin{array}{l}
u_{i}^{t} \\
h_{i}^{t}
\end{array}\right)+\frac{\triangle t}{2}\left[\mathbf{F}\left(u_{i}^{t}, h_{i}^{t}\right)+\overline{\mathbf{F}}\left(\overline{u_{i}^{t+1}}, \overline{h_{i}^{t+1}}\right)\right],
$$

where $\overline{u_{i}^{t+1}}$ and $\overline{h_{i}^{t+1}}$ are "provisional" values at time level $t+1$, and $\mathbf{F}$ represents all the partial spatial derivative terms on the right-hand side (expressions for $\mathbf{F}$ are listed in Appendix C).

\section{Time-step and grid-size convergence}

To test the integrity of the SLE numerical approach introduced above, we consider the simulation of a short thread $(L=10, \mathrm{Oh}=1.07$, and $\mathrm{Th}=0.11)$ with an increasingly fine time step and grid spacing (note, for this case, a model using uncorrelated noise would not converge).

We set dimensional $\tilde{T}_{\mathrm{c}}=0.01 \mathrm{ps}$ and $\tilde{L}_{\mathrm{c}}=0.5 \mathrm{~nm}$ for all the simulations presented in this article (corresponding to dimensionless $T_{\mathrm{c}}=4.66 \times 10^{-5}$ and $L_{\mathrm{c}}=0.2$ ); chosen to be similar to that seen in our MD data. Notably, since this is a stochastic system, it is the convergence of the ensembleaveraged quantities that we are concerned with; here the ensemble consists of 100 independent simulations.

The ensemble-averaged interface profiles at two-time instances are plotted in Fig. 4(a) for varying grid size and Fig. 4(b) for varying time step. Note, in this paper we plot all interface and rupture profiles relative to the minimum point (i.e., we plot $h$ against $z-z_{\min }$, where $z_{\min }(t)$ is the location 

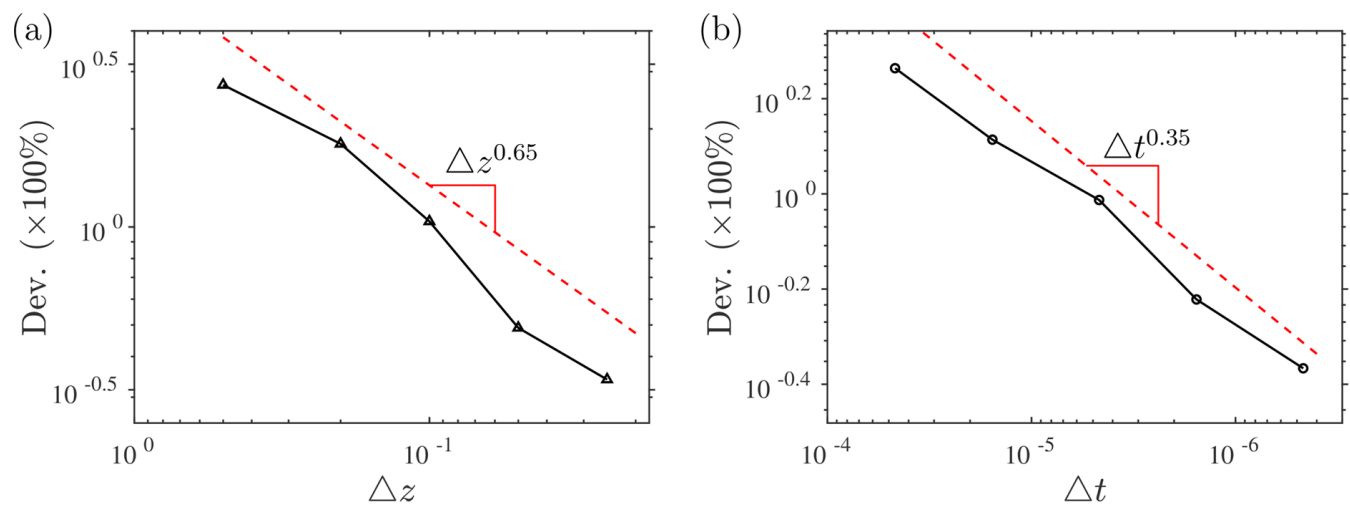

FIG. 5. Convergence characteristics for decreasing (a) grid size and (b) time step. Average (over $z$ ) deviation of ensemble-averaged interface profiles to the finest resolution profile in Figs. 4(a) and 4(b), respectively.

of the minimum in thread radius at any instant in time). To better demonstrate the convergence of the method, we calculate the average deviation of each ensemble-averaged profile to that with the finest resolution calculated; Fig. 5 confirms that this deviation steadily decreases (albeit slowly) with increasing [Fig. 5(a)] spatial and [Fig. 5(b)] temporal resolution (i.e., it converges).

\section{NUMERICAL VERIFICATION AND VALIDATION}

Having demonstrated the convergence, the numerical solutions are verified and validated by the analytical models and MD simulations at both the linear stage for instability (Sec. III A) and the nonlinear stage for rupture (Sec. III B).

\section{A. Linear instability and thermal capillary waves}

In this section, the analytical SLE-RP [24] has been employed as a benchmark for the numerical solutions of the SLE introduced above (Sec. II C). The SLE-RP can be written in a dimensionless form as follows (see Appendix D for derivation):

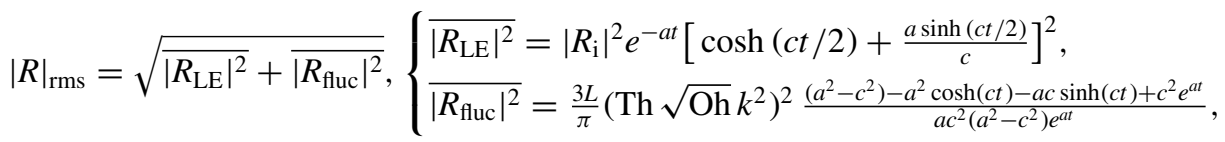

where

$$
a=3 \mathrm{Oh} k^{2} \text { and } c=\sqrt{\left(9 \mathrm{Oh}^{2}-2\right) k^{4}+2 k^{2}} .
$$

Here, $k$ is the dimensionless wave number, $L$ is the dimensionless length of the thread, and $R_{\mathrm{i}}$ is the initial model disturbance (a complex random variable with zero mean).

We also perform MD simulations for comparison, where we adopt a coarse-grain water molecule model, $\mathrm{mW}$ [40], to limit the computational resources required for such long threads; in all cases, $L=100$. The inital radius $r_{0}$ and temperature $T$ is selected to obtain specific Oh and Th (shown in Table I): Threads 1 and 2 have the same Th; Threads 2 and 3 have the same Oh.

For each case we extract statistics from an ensemble of independent simulations (or "realizations"); 20 for MD and 50 for the SLE (true for the rest of the paper, unless otherwise stated). For each realization, a discrete Fourier transform of the interface position (which in MD is extracted from axially distributed annular bins based on a threshold density) is applied to get the power spectral density (PSD). The square root of the ensemble-averaged PSD at each time is plotted in 
TABLE I. Case setups of mW.

\begin{tabular}{lcccccccc}
\hline \hline Thread & $r_{0}(\mathrm{~nm})$ & $T(\mathrm{~K})$ & $\gamma\left(\mathrm{N} \mathrm{m}^{-1}\right)$ & $\rho\left(\mathrm{kg} \mathrm{m}^{-3}\right)$ & $\mu\left(\mathrm{kg} \mathrm{m}^{-1} \mathrm{~s}^{-1}\right)$ & Oh & Th & Number of molecules \\
\hline$(1)$ & 2.410 & 275.6 & $6.53 \times 10^{-2}$ & $1.006 \times 10^{3}$ & $3.582 \times 10^{-4}$ & 0.90 & 0.10 & 147,828 \\
$(2)$ & 2.891 & 354.8 & $5.85 \times 10^{-2}$ & $0.988 \times 10^{3}$ & $2.043 \times 10^{-4}$ & 0.50 & 0.10 & 250,484 \\
$(3)$ & 5.170 & 304.4 & $6.29 \times 10^{-2}$ & $1.001 \times 10^{3}$ & $2.851 \times 10^{-4}$ & 0.50 & 0.05 & $1,451,568$ \\
\hline \hline
\end{tabular}

Fig. 6 and compared to the SLE-RP [Eq. (11)]. The agreement between both numerical results and the analytical ones is very good for each case and at each time, giving us further confidence that the SLE implementation is both numerically sound and capable of capturing nanoscale flow physics (as demonstrated in Ref. [24], where we used a Lennard-Jones potential).

The results in Fig. 6(a) show a modal distribution (spectrum) that varies with time. For small wave numbers $(k<1)$, the spectrum becomes sharper with time, while the spectrum at high wave numbers $(k>\sim 2)$ is static over these timescales; i.e., it quickly reaches its asymptotic limit. This limit can be obtained from Eq. (11), for $k>1$, by taking $t \rightarrow \infty$ :

$$
|R|_{\mathrm{rms}}=\sqrt{\frac{L}{2 \pi}} \mathrm{Th} \sqrt{\frac{1}{k^{2}-1}} .
$$

This is consistent with the theory for thermal capillary waves in thin-film flows [46,47], which describes the time-invariant state of a liquid interface by a balance between capillary forces (surface tension) and thermal fluctuations. The crucial difference to thin-film flows is seen at small wavenumber perturbations, which are unstable for the liquid thread because of the surface tension component acting around the thread's circumference - this is the Rayleigh-Plateau instability. Interestingly, Eq. (12) indicates that the asymptotic limit for $k>1$ (i.e., the part of the spectrum composed of thermal capillary waves) only depends on Th; as confirmed in Fig. 6(b), where Threads 1
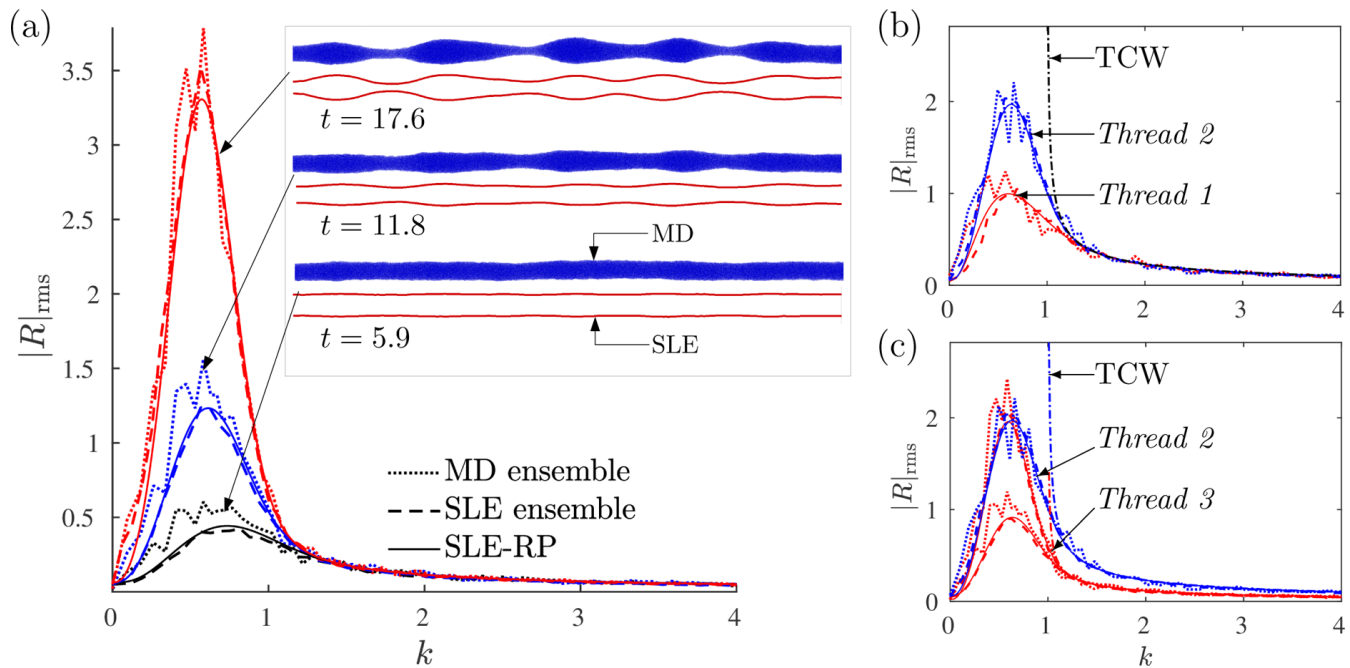

FIG. 6. The root mean square (rms) of nondimensional disturbance amplitude versus nondimensional wave number; a comparison of ensemble-averaged MD simulations (dotted lines), ensemble-averaged SLE simulations (dashed lines), the SLE-RP analytical result (solid lines), and thermal capillary wave (TCW) theory (dashed-and-dotted lines). Comparisons are for (a) Thread 3 at three (nondimensional) time instances, $t=5.9$, 11.8, and 17.6; (b) Threads 1 and 2 (equal Th) for $t=9.3$; and (c) Threads $2(t=9.3)$ and $3(t=9.3,14.7)$ with the equal Oh. The inset in (a) shows selected MD and SLE realizations. 
TABLE II. Case setups of TIP4P/2005.

\begin{tabular}{lcccccccc}
\hline \hline Thread & $r_{0}(\mathrm{~nm})$ & $T(\mathrm{~K})$ & $\gamma\left(\mathrm{N} \mathrm{m}^{-1}\right)$ & $\rho\left(\mathrm{kg} \mathrm{m}^{-3}\right)$ & $\mu\left(\mathrm{kg} \mathrm{m}^{-1} \mathrm{~s}^{-1}\right)$ & Oh & Th & Total molecules \\
\hline$(4)$ & 2.020 & 358.1 & $5.38 \times 10^{-2}$ & $0.964 \times 10^{3}$ & $3.230 \times 10^{-4}$ & 1.00 & 0.15 & 10,246 \\
$(5)$ & 2.675 & 316.6 & $6.09 \times 10^{-2}$ & $0.987 \times 10^{3}$ & $5.827 \times 10^{-4}$ & 1.45 & 0.10 & 23,791 \\
\hline \hline
\end{tabular}

and 2 have the same Th and the same spectrum at high wave numbers. Figure 6(c) shows that the spectrum with the larger Th (Thread 2) is broader (at the same nondimensional instant): stronger thermal fluctuations lead to a wider distribution of wave numbers. Since the droplet sizes are related to the dominant wave numbers, we can expect a broader and enhanced distribution of the probability density function for droplet sizes with larger Th. This hypothesis is supported by results from a fluctuating lattice Boltzmann model in Ref. [48].

\section{B. Rupture dynamics}

In this section, numerical solutions to the fully nonlinear SLE are compared to MD simulations for rupture dynamics. For the MD in this section, the TIP4P/2005 water model [41] is adopted, with liquid properties as listed in Table II. Here, $L=12$.

Our first comparison, Fig. 7, is for the time evolution of the minimum (over $z$ ) thread radius, $h_{\min }(t)$. Since our focus here is on the dynamics near rupture, $h_{\min }$ is plotted against time to rupture, $t_{\mathrm{b}}-t$, where $t_{\mathrm{b}}$ is the time at rupture. The red error bars and shadows represent one standard deviation (either side of the mean) for the MD and the SLE, respectively. In the two cases (a) Thread 4 and (b) Thread 5, good agreement is found at all times for the mean, but also, importantly, for the standard deviation.

Figure 7 suggests that a power law might govern the progression of the minimum thread thickness to rupture: $h_{\min } \propto\left(t_{\mathrm{b}}-t\right)^{\alpha}$. However, despite exhibiting a power law, these results are not described well by the similarity solution proposed by Eggers [25] for which the exponent $\alpha=0.418$. One possible reason for the discrepancy is that Eggers neglected the influence of surface tension. To explore this explanation, we can exploit the SLE numerical model-MD is unable to perform such simulations due to inherent restrictions on the variation of liquid properties. We test different values for surface tension with all other parameters (i.e., $\rho, \mu$, and $T$ ) fixed. The average $h_{\min }(t)$ obtained from 50 realizations is plotted on a logarithmic scale in Fig. 8. The results indicate that the numerical solutions (solid lines) do tend towards Eggers' similarity solution (red dashed lines) for lower values of surface tension (note we vary a dimensional quantity here, to connect most transparently with the
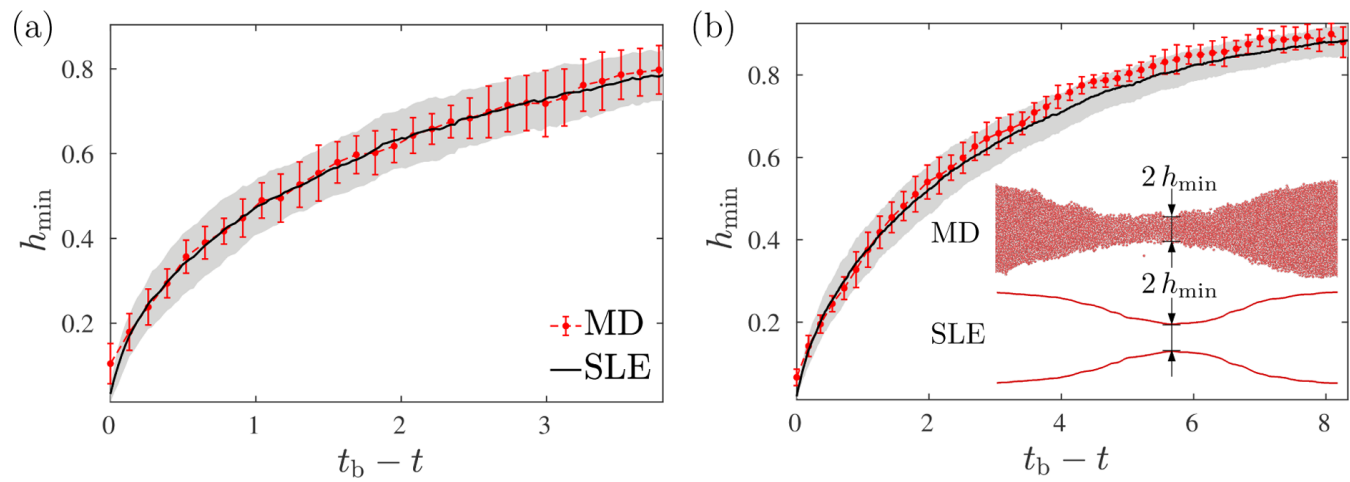

FIG. 7. Minimum thread radius against time to rupture $\left(t_{\mathrm{b}}-t\right)$. Comparison of MD and nonlinear SLE for (a) Thread 4 and (b) Thread 5 . 


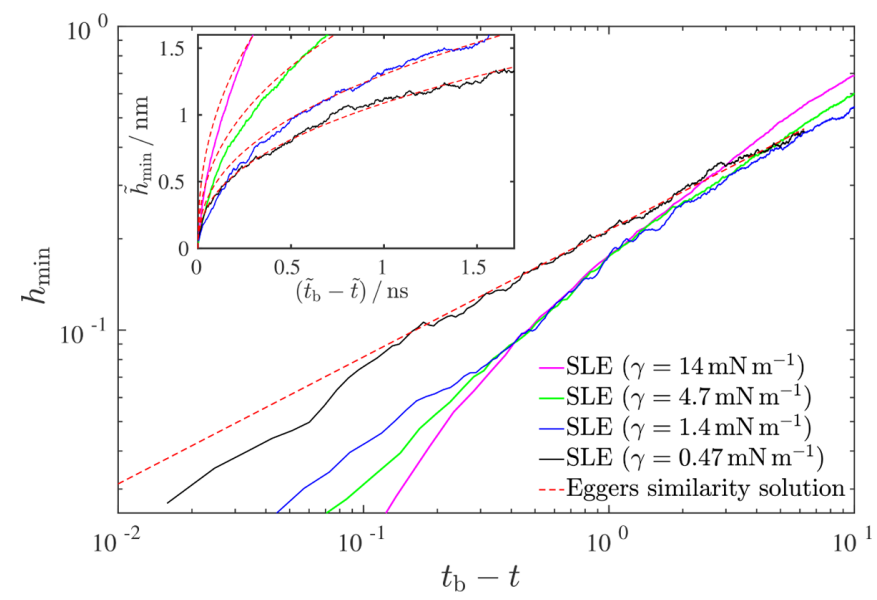

FIG. 8. The temporal evolution of the minimum thread radius for different values of surface tension: comparison between similarity solutions [25] and an ensemble-average of nonlinear SLE calculations. Here, the choice of the maximum value of the surface tension $\left(\gamma=14.0 \mathrm{mN} \mathrm{m}^{-1}\right)$ used in the SLE comes from liquid argon at $84.0 \mathrm{~K}$.

assumption in Eggers' work). Notably, when surface tension is stronger, the breakup is faster than the analytical prediction, which suggests that the destabilising effect of surface tension can also contribute to the thread dynamics near to rupture. This limit of applicability might also explain the deviation between Eggers' similarity solution and MD results in previous studies [49,50].

Although the agreement for $h_{\min }$ with Eggers' similarity solution is good for low $\gamma$, we were unable to find any agreement between either MD or SLE with the associated universal profile. The reason is currently unclear and should be the subject of future investigation.

The ensemble-averaged profiles plotted in Fig. 9 show good overall agreement between the MD and SLE for three time instances leading to rupture. The limitation of bin sizes in the MD data prevents a more detailed comparison of the profile shape. In particular, it is not clear whether the finer features seen in the SLE (namely, the V-notch—or "widow's peak"-near to the minimum) is physical because these local features reach the molecular scale and cannot be reliably extracted from the MD.
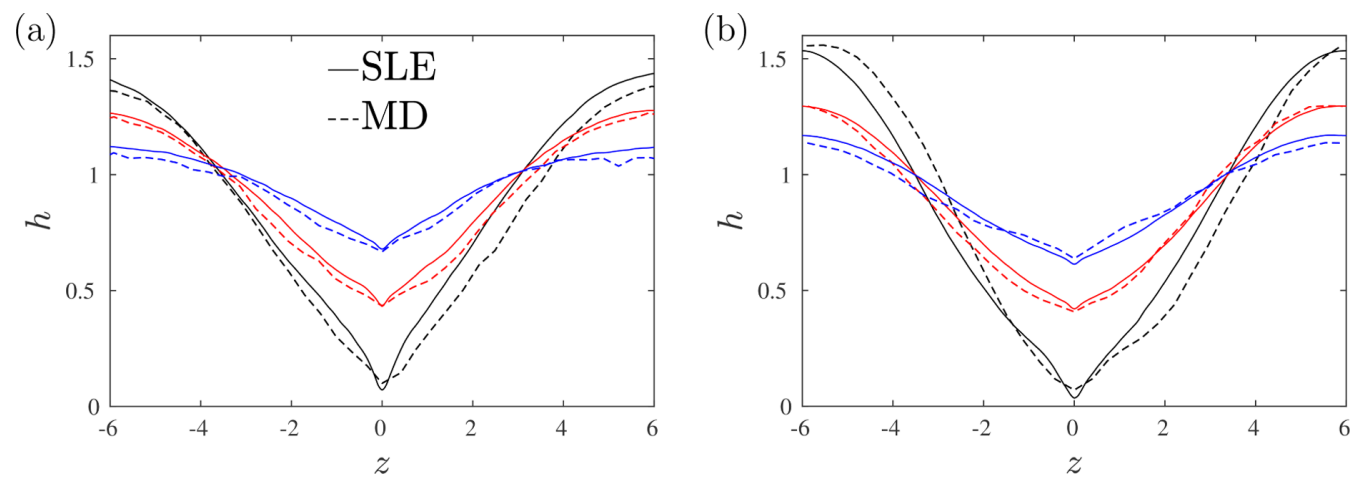

FIG. 9. Ensemble-averaged interface profiles at three time instances leading to rupture: a comparison of the nonlinear SLE solver (solid lines) and MD data (dashed lines); (a) Thread 4: $t_{\mathrm{b}}-t_{1}=0.13$ (black), $t_{\mathrm{b}}-$ $t_{2}=1.04$ (red), $t_{\mathrm{b}}-t_{3}=2.73$ (blue); (b) Thread 5: $t_{\mathrm{b}}-t_{1}=0.09$ (black), $t_{\mathrm{b}}-t_{2}=1.44$ (red), $t_{\mathrm{b}}-t_{3}=3.50$ (blue). 

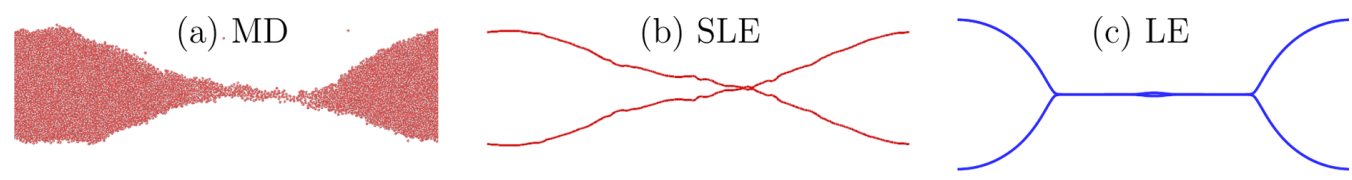

FIG. 10. Thread 5 rupture profiles from different models: (a) an MD simulation (b) a selected realization from the SLE (c) a solution to the LE (conventional lubrication equations).

\section{EXPLOITING SLE: RUPTURE BEYOND MD}

Having established its predictive capability, in this section we use the nonlinear SLE solver to further explore the impact of fluctuations on rupture dynamics, over a broader range of conditions than have been studied previously and for cases that are too computationally demanding to consider with MD. We start, in Sec. IV A, by exploring the shape of the thread at rupture whilst in Sec. IV B, we focus on the time evolution of the point of the thread's minimum thickness.

\section{A. Rupture profiles}

Moseler and Landmann [18] were the first to demonstrate, using MD, that thermal fluctuations could lead to a symmetric double-cone rupture profile, and that SLE solutions were also able to capture this (whereas deterministic equations cannot). We reproduce this result for Thread 5 (see Table II for parameters) in Fig. 10. Note that the nonlinear SLE solution can reproduce the MD result, where the (deterministic) LE cannot, at a fraction of the computational cost of MD. Here, each MD realization of Thread 5 needs about 4600 core hours, while one SLE solution (the finest resolution) costs less than 1 core hour; i.e., the speed-up is about $10^{3}$.

These computational advantages allow the SLE to be applied in a far broader range of conditions than accessible to MD; as is illustrated in Fig. 11.

Figure 11(a) shows a rupture profile from a macroscopic experiment [51] (with very small Th), which exhibits a satellite droplet between two main drops. This macroscale structure can be captured by the SLE solution (blue lines), since it reverts to the classical LE solution as $\mathrm{Th} \rightarrow 0$. The rupture profile in Fig. 11(b) is from an experiment at several microns, where a colloid-polymer mixture is used to make Th larger, not to generate a nanoscale thread. While the profile here is not a macroscopic one, it cannot be well described as a pure double-cone rupture either. However the SLE can faithfully reproduce such shapes, which are associated with intermediate Th (moderate fluctuations). Figure 11(d) shows that the SLE solution can also capture a pure double-cone profile with a large Th at the nanoscale where only MD experiments are currently available for comparison.

Importantly, however, as done in Ref. [18], all the rupture profiles above are selected realizations. In other words they have been picked, from numerous independent SLE results, based on their qualitative similarity to the experimental or MD result being compared to. From these selections, then, it is not possible to ascertain whether the SLE has captured the full dynamics of the rupture, which is of course statistical in nature. To do this we must compare, at the least, the expected and/or most-probable profile, and some measure of the statistical fluctuation. Establishing such a framework is one of the main contributions of this paper.

Figure 12 shows a matrix of profiles for varying Th and Oh, obtained using the periodic boundary conditions. For comparison, the left-hand column $(\mathrm{Th}=0)$ contains the rupture profiles as predicted by classical LE. Note that there are two rupture points at $\mathrm{Oh}=0.02$ and 1 , due to the satellite drop. For consistency, we select the left one and move it to the center $(z=0)$. For the stochastic results $(\mathrm{Th}>0)$, each realization is centered on its rupture point (i.e., the rupture is located at $z=0$ ). To preserve large anti-symmetrical features, that would otherwise be averaged out, each centered profile is flipped about $z=0$ so that $\int_{-L / 2}^{0} h \mathrm{~d} z>\int_{0}^{L / 2} h \mathrm{~d} z$. In the figures, the solid blue lines are the ensemble-averaged rupture profiles (averaged after the centering and transformation described above). Gray shading indicates the region between the 10th and 90th percentile value of 
Experiments/MD

(a)

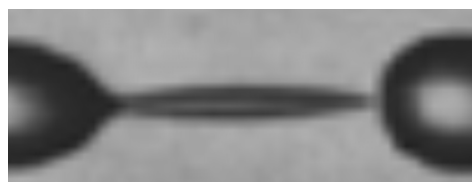

(b)

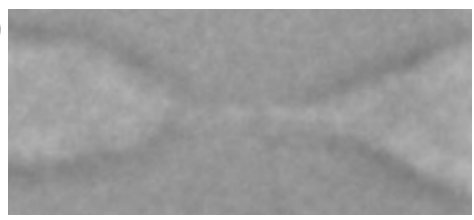

(c)

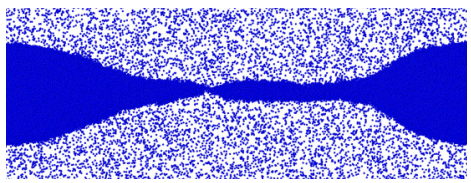

(d)

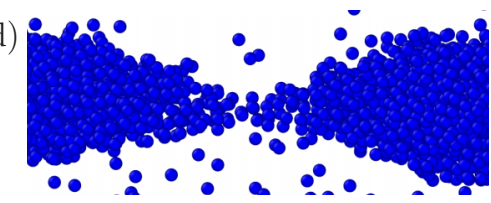

SLE

Scale

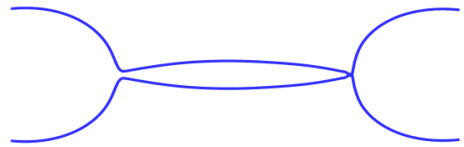

Millimetre

$r_{0}=4.00 \mathrm{~mm}$

Micrometre

$r_{0}=6.00 \mu \mathrm{m}$

Nanometre $r_{0}=5.76 \mathrm{~nm}$

Nanometre $r_{0}=1.44 \mathrm{~nm}$

FIG. 11. Comparison of rupture profiles in experiments (a), (b) and MD (c), (d) with numerical selected solutions to the SLE: (a) $\mathrm{Oh}=2.60 \times 10^{-3}$, Th $=1.17 \times 10^{-7}$, experimental image reproduced from [51]; (b) $\mathrm{Oh}=1.00, \mathrm{Th}=4.50 \times 10^{-2}$, experimental image reproduced from [19]; (c) $\mathrm{Oh}=0.71$, $\mathrm{Th}=4.96 \times$ $10^{-2}$, Cylinder 1 from Ref. [24]; and (d) $\mathrm{Oh}=1.41$, Th $=1.98 \times 10^{-1}$, Cylinder 3 from Ref. [24].

$h$ (determined at each $z$ ); i.e., for a given $z$ we can be $80 \%$ confident that the profile exists within it. Note, as $h$ is always greater than zero, the distribution of its value is not Gaussian, hence the mean/expected profile is not necessarily the same as the most probable. We can crudely approximate the positive distribution of $h$ at any point in $z$ with a Gamma distribution, and from that a mostprobable profile (from the peak of the distribution at each $z$ ) can be estimated (the red dashed lines). Notably, it is the most probable profile which Eggers [25] computes from the Fokker-Planck equation for the SLE. In the cases considered here there is little difference between the mean and the estimated most-probable profile.

The bottom right-hand corner profile in Fig. 12 qualitatively reproduces the findings of Moseler and Landman [18] and Eggers [25]: a largely symmetric double-cone profile is observed, although it appears this may be better described as an "hourglass." One might naively expect that the importance of fluctuations on thread dynamics would be solely determined by the value of Th (the ratio of the thermal fluctuation scale to the thread radius). However, what is striking is that neither the relative magnitude of fluctuations (the shaded regions) or the impact of noise on the mean profile is dictated by $\mathrm{Th}$ alone. For example, at $\mathrm{Th}=0.02$, the influence of fluctuations on the dynamics can either be negligible or profound, depending on Oh. Nor is it easy to identify a combination of Oh and Th [e.g., Th $\sqrt{\mathrm{Oh}}$ from Eq. (4)] that might be useful in singularly describing when fluctuations become important or not: it is, seemingly, a nontrivial interplay of effects as we would expect when inertia, viscosity, surface tension, and fluctuations all play a role.

The next most important observation is that, for low $\mathrm{Oh}$, the impact of noise results in an asymmetric mean thread profile at rupture (see top right-hand corner image of Fig. 12). The double-cone profile observed in Moseler and Landman [18] is not observed here. Instead, we see a quite distinct rupture shape (a drop and funnel), on average, which looks more like typical 

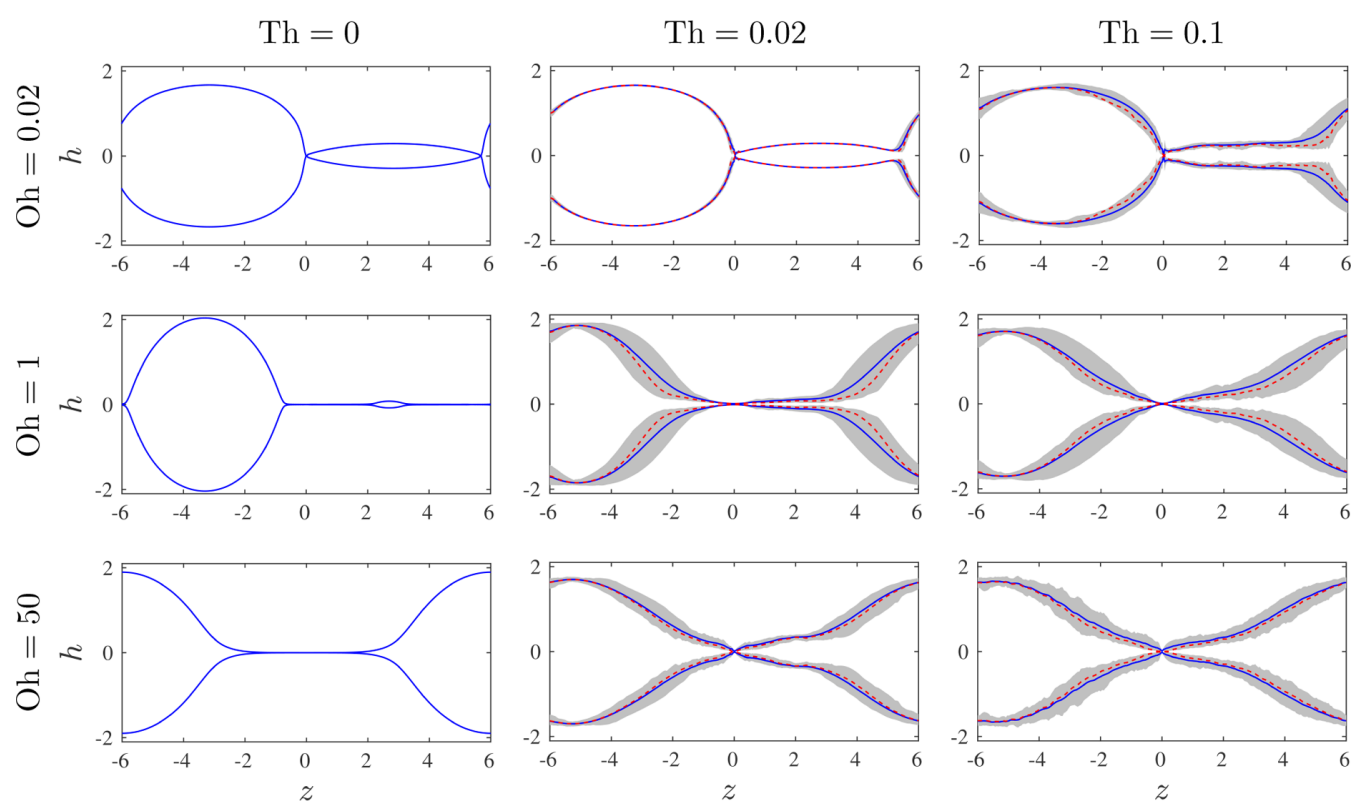

FIG. 12. Rupture profiles for different combinations of Oh and Th. Solid lines represent the ensemble average (the expected profile), dashed lines an estimate of the most-probable profile, and the region bounded by the 10th and 90th percentile value of $h(z)$ is shaded gray.

rupture profiles seen macroscopically when a drop breaks off from a thread. This behavior is not surprising, as the chance of two points pinching off at precisely the same instance becomes slim when we have fluctuations (and indeed this kind of "perfect" pinch off is difficult to reproduce experimentally macroscopically as well). We stress that these flow conditions are not accessible by our MD simulations at present; the SLE calculations are essential to provide this insight.

\section{B. Evolution of minimum thread radius}

In the classical picture there is the potential for multiple transitions between distinct "dynamic regimes" (defined by Oh) leading to rupture [7,13,14]. The three main regimes, described in Sec. I, are the viscous regime (V-regime), the inertial regime (I-regime), and the universal viscous-inertial regime (VI-regime). These regimes are characterised by a power-law (linear for the first and third) evolution of minimum thickness with time to rupture, at rates given by various analytical results $[7,13]$.

On top of this already complex situation, thermal fluctuations can introduce yet another regime (here referred to as the F-regime), which generates nonlinear (power-law) evolution of minimum thread radius. For moderate Th $(>\sim 0.1)$ and nonnegligible Oh, fluctuations appear to dominate the entire thread evolution [see, e.g., the nonlinear evolution in Fig. 7(a)]. However, at lower Th, we can observe transitions from the classical behavior to one that is fluctuation dominated as the rupture process progresses.

In Fig. 13 we compare the SLE with the classical model (LE) and various analytical results for fixed $\mathrm{Th}=0.02$ at $\mathrm{Oh}=0.01,1$ and 100. Experimentally, this corresponds to using fluids of a range of different viscosities (with fixed surface tensions) for the same breakup configuration. As was the case for the rupture profiles presented above, at low Oh [Fig. 13(a)] there is seemingly no impact of fluctuations on the time evolution of $h_{\min }$ (i.e., there is no discernible difference between the SLE and LE). For larger Oh [see Fig. 13(c)], however, a clear transition between macroscopic and a fluctuation-dominated regime can be observed. At early times the evolution is described by 

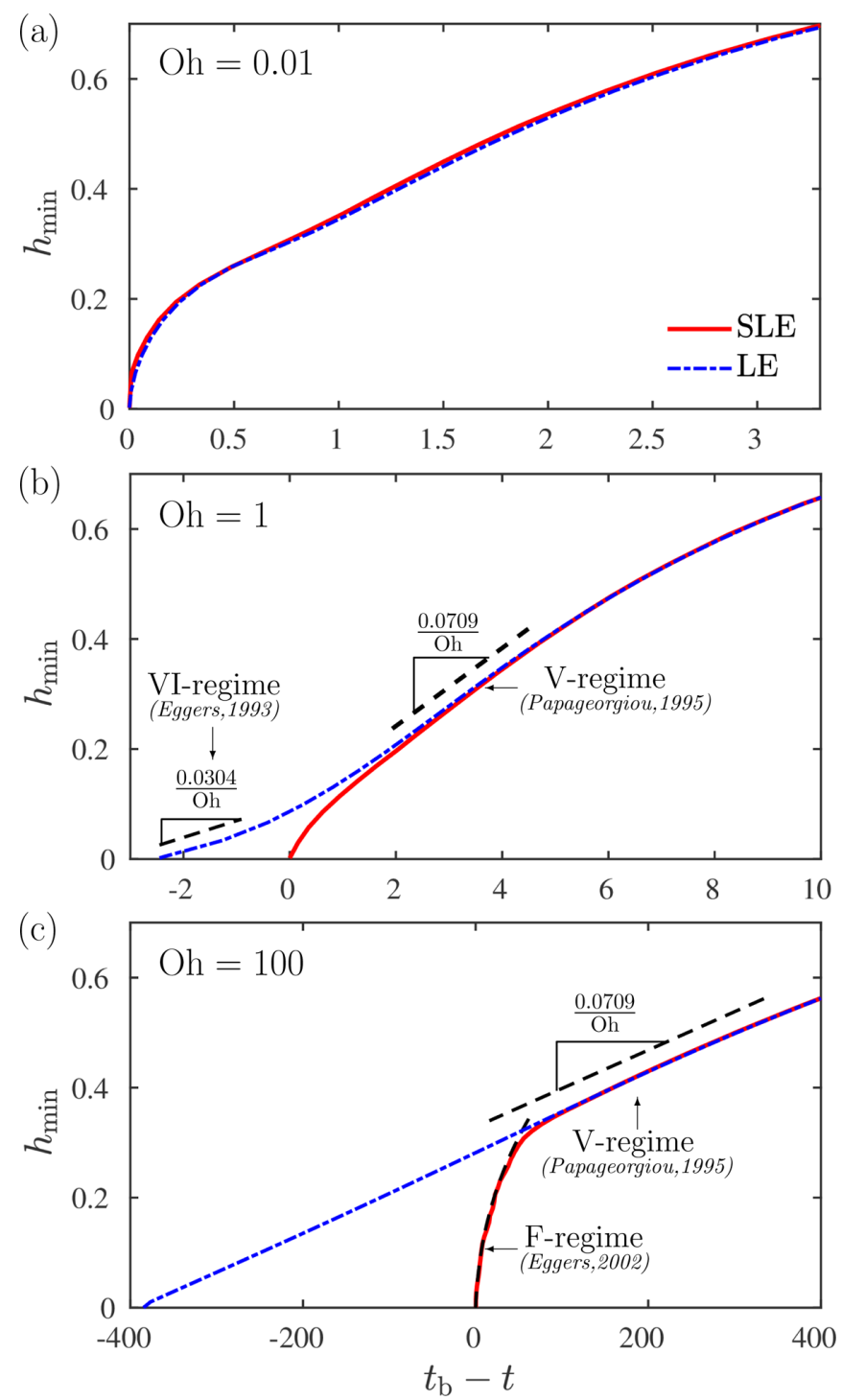

FIG. 13. Time evolution of minimum thread radius, $h_{\min }$; a comparison between the LE (dashed and dotted lines) and SLE (solid lines) for $\mathrm{Th}=0.02$ and $\mathrm{Oh}=$ (a) 0.01 , (b) 1 , and (c) 100. Note, $t_{\mathrm{b}}$ is the breakup time predicted by the SLE. Dashed lines represent similarity solutions in different dynamic regimes. Linear solutions in the VI-regime and V-regime come from Refs. [12] and [10], respectively. The power-law solution in the F-regime for (c) is obtained from Ref. [25].

a linear time dependence, derived by Papageourgiou [10,11] for the V-regime; at later times (in the F-regime) the evolution matches the power law proposed by Eggers [25] and greatly accelerates the breakup process. Of course, of the two numerical methods presented in the figure, only the SLE can capture both. When $\mathrm{Oh}=1$ [Fig. 13(b)], the dynamics become more complicated. The LE predicts a transition from the V-regime to the VI-regime in the final stages, which has been proved experimentally [13] and numerically [7] at macroscopic scales. However, this transition does not occur in the presence of thermal fluctuations, according to the SLE. Instead there exists a similar transition from the V-regime to a new regime as was the case for the large Oh case, but the power-law 


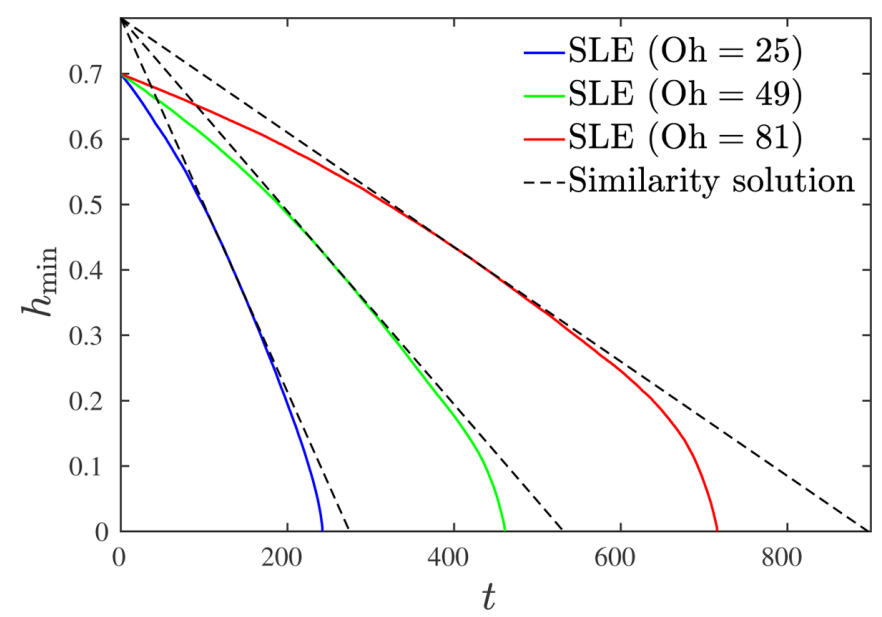

FIG. 14. The temporal evolution of minimum thread radius for different $\mathrm{Oh}$, with $\mathrm{Th}=0.02$. The similarity solution comes from the Ref. [11].

exponent does not match that found by Eggers (which, as explored in Sec. III, is possibly due to the assumption in his analytic treatment that surface tension is unimportant in the final stages leading to rupture).

Figure 14 shows more results of the transition from the V-regime to the "fluctuation" regime, where the dashed lines are Papageorgiou's similarity solution for different $\mathrm{Oh}$ and solid lines represent the average solutions (from 50 realizations) for the SLE. These results indicate a bigger "fluctuation" regime (larger crossover $h_{\min }$ ) with larger Oh, highlighting the important role of Oh on the fluctuation intensity. Furthermore, it would be interesting to explore whether there exists a scaling law between the height at which this transition occurs and Oh (or Th). However, we would need to get many decades of $h_{\min }$ to determine the precise crossover point, which is not available from our current simulations.

\section{DISCUSSION AND FUTURE DIRECTIONS}

In this work, a numerical solver of the SLE has been developed with a new simple scheme proposed for the noise term. Based on validation from MD for both instability and the rupture of liquid nanothreads, this solver is demonstrated to be a powerful tool for studying the interface dynamics of nanothreads; and operating over a thousand times faster than MD. Furthermore, it allows us to operate in the regions of parameter space where analytic models are outside their limits of applicability and MD is impractical either due to (i) exorbitant computational cost or (ii) limits in the molecular properties available from known potentials.

While this article provides new understanding of interface dynamics, it opens up several new avenues of enquiry, that we discuss here.

\section{A. Correlation scales}

In this article the use of a correlation scale is motivated from two angles. First, the computational SLE scheme is seen to be unable to converge unless these are introduced, with huge spikes on the free surface observed that seemingly prematurely rupture the thread and/or destroy the numerical accuracy. Second, MD suggests that correlation scales exist, and as one may expect these are typically on the molecular scale. These issues motivate a number of different questions. From a modeling viewpoint, the incorporation of molecular correlation scales within a continuum model should be treated with caution, and thus one may interpret a continuum limit as when the correlation 
length goes to zero. However, in some cases the correlations seem to have a profound effect on thin film dynamics simulations and experiments [28], so that these issues are far from trivial. In terms of numerical analysis, there are interesting questions regarding convergence, both as one considers the mesh size/time step going to zero and as the correlation scale becomes small. For example, what should we expect if we consider a fixed number of cells within each correlation scale and then take the correlation scale to zero (as one may expect for the continuum limit)? Such questions are related to the development of robust and efficient numerical schemes for SPDE problems. Here, we focus on simplicity, both with the use of an explicit time-stepping scheme and in the linear interpolation of noise. More complex schemes exist, where the noise is represented in terms of appropriate basis functions [26,29], but although these are more mathematically rigorous approaches, we found them to give the same dynamic behaviours at increased computational cost. Clearly there is scope for more work in this direction, particularly as one considers the possibility of developing 2D schemes, as even though the SLE is much cheaper than MD, it is still more computationally burdensome than deterministic methods (as a minimum, due to the requirement for ensembles).

\section{B. Similarity solutions}

By considering a liquid with sufficiently small surface tension, we were able to recover the power law predicted by Eggers' similarity solution [25], as previously also identified in specially designed experiments with colloid-polymer mixtures [19]. However, no agreement was obtained between the SLE and similarity solution for the universal profiles predicted in Ref. [25], and surface tension was seen to influence the power law even at physical values, as seen in Figs. 8 and 13. Therefore, it remains an open problem to derive similarity solutions for the breakup that incorporate surface tension, building on the new framework, considering the most probable breakup in a stochastic process, as developed by Eggers for this class of flows.

\section{Transition prediction}

Liquid thread rupture is a multiscale phenomenon with complicated transitions between different regimes. Numerical solutions in Figs. 13 and 14 show a transition from the V-regime to fluctuationdominated regimes. However, it remains unclear whether there exists a scaling law between the crossover (transition) point and Oh. To answer this question, we need to develop more accurate and efficient schemes (e.g., higher-order schemes and implicit time marching methods) to capture many decades of dynamics, which could be the subject of future work.

\section{Experimental analysis}

In nanoscale breakup phenomena, one not only has small spatial scales but also small temporal ones for the problems of interest. This is in contrast to thin film dynamics, where typical timescales are macroscopic when highly viscous films are considered [52,53] and therefore experimental analysis that temporally resolves features becomes possible. For the problems considered in this article, it seems most likely that experimental verification would come first from the ultralow surface tension liquids developed in Refs. $[19,20]$ that make Th moderate even at the microscale where one can perform imaging. There are many potential directions for experimental analysis to take, but a starting point would be to more carefully consider the rupture profiles and scaling of $h_{\min }$ to see how this compares to our predictions.

\section{ACKNOWLEDGMENTS}

This work was supported by the Leverhulme Trust (Research Project Grant) and the EPSRC (Grants No. EP/N016602/1, No. EP/P020887/1, and No. EP/P031684/1). 
TABLE III. Parameters of the mW model.

\begin{tabular}{lccccccccc}
\hline \hline$\epsilon\left(\mathrm{kJ} \mathrm{mol}^{-1}\right)$ & $\sigma(\mathrm{nm})$ & $A$ & $B$ & $p$ & $q$ & $\chi$ & $\kappa$ & $a$ & $\theta_{0}$ (degree) \\
\hline 25.87 & 0.2390 & 7.050 & 0.6022 & 4 & 0 & 1.2 & 23.15 & 1.8 & 109.47 \\
\hline \hline
\end{tabular}

\section{APPENDIX A: MOLECULAR MODELS}

In this section, we introduce the two molecular models used in this work: (i) $\mathrm{mW}$ [40] and (ii) TIP4P/2005 [41].

In $\mathrm{mW}$ model, the hydrogen-bonded structure of water is mimicked through the introduction of a nonbond angular dependent term that encourages tetrahedral configurations. The model contains two terms: (i) $\phi_{i j}$ depending on the distances between pairs of atoms (represented by $r_{i j}$ and $s_{i k}$ ); and (ii) $\phi_{i j k}$ depending on the angles formed by triplets of atoms (represented by $\theta_{i j k}$ ). The full expression is given by

$$
\begin{aligned}
U & =\sum_{i} \sum_{j>i} \phi_{i j}\left(r_{i j}\right)+\sum_{i} \sum_{j \neq i} \sum_{k>j} \phi_{i j k}\left(r_{i j}, s_{i k}, \theta_{i j k}\right), \\
\phi_{i j}\left(r_{i j}\right) & =A \epsilon\left[B\left(\frac{\sigma}{r_{i j}}\right)^{p}-\left(\frac{\sigma}{r_{i j}}\right)^{q}\right] \exp \left(\frac{\sigma}{r_{i j}-a \sigma}\right), \\
\phi_{i j k}\left(r_{i j}, s_{i k}, \theta_{i j k}\right) & =\kappa \epsilon\left(\cos \theta_{i j k}-\cos \theta_{0}\right)^{2} \exp \left(\frac{\chi \sigma}{r_{i j}-a \sigma}\right) \exp \left(\frac{\chi \sigma}{s_{i k}-a \sigma}\right),
\end{aligned}
$$

where $\epsilon$ is the depth of the potential well, $\sigma$ represents the particle diameter, $A, B, p, q, \chi$, and $\kappa$, respectively, give the form and scale to the potential, and $\theta_{0}$ represents the tetrahedral angles. All the parameters are presented in Table III.

The TIP4P/2005 water model [41] is constructed based on the Bernal-Fowler geometry with four parts: an oxygen atom $(\mathrm{O})$ with no charge, two hydrogen atoms $(\mathrm{H})$ with a point charge, and a massless part (M) with charge. According to experimental data, the $\mathrm{O}-\mathrm{H}$ distance and $\mathrm{H}-\mathrm{O}-\mathrm{H}$ angle are set as $0.9572 \AA$ and $104.52^{\circ}$, respectively. The intermolecular pair potential can be divided into two different types: (i) the Lennard-Jones (LJ) potential and (ii) the Coulomb (electrostatic) potential. So the expression for the pair potential is

$$
U\left(r_{i j}\right)=4 \epsilon\left[\left(\frac{\sigma}{r_{i j}}\right)^{12}-\left(\frac{\sigma}{r_{i j}}\right)^{6}\right]+\frac{1}{4 \pi \varepsilon_{0}} \frac{q_{i} q_{j}}{r_{i j}},
$$

where $\varepsilon_{0}$ represents the vacuum permittivity, $q_{i}$ and $q_{j}$ are the atomic charges. We list all the parameters for different atoms in Table IV.

\section{APPENDIX B: DERIVATION FOR THE NOISE VARIANCE}

In this section, a mathematical procedure is proposed to derive the variance of our numerical correlated noise model in the main text. To simplify the problem further, we only use temporal

TABLE IV. Parameters of the TIP4P/2005 model.

\begin{tabular}{lclc}
\hline \hline Atom & $\epsilon\left(\mathrm{kJ} \mathrm{mol}^{-1}\right)$ & $\sigma(\mathrm{nm})$ & $q(e)$ \\
\hline $\mathrm{H}$ & 0 & 0 & 0.5564 \\
$\mathrm{O}$ & 0.775 & 0.315 & 0 \\
$\mathrm{M}$ & 0 & 0 & -1.112 \\
\hline \hline
\end{tabular}


fluctuations in this example. The variance of the fluctuation is

$$
\left\langle N(t) N\left(t^{\prime}\right)\right\rangle=A_{\mathrm{f}}^{2} \delta\left(t-t^{\prime}\right),
$$

where $A_{\mathrm{f}}$ is the noise amplitude. The mean of the noise, $N$, over a period $T_{N}$ is

$$
\bar{N}=\frac{1}{T_{N}} \int_{0}^{T_{N}} N(t) d t,
$$

where the variance of $\bar{N}$ can be obtained by

$$
\begin{aligned}
\left\langle\frac{\int_{0}^{T_{N}} N(t) d t}{T_{N}} \frac{\int_{0}^{T_{N}} N\left(t^{\prime}\right) d t^{\prime}}{T_{N}}\right\rangle & =\frac{1}{T_{N}^{2}} \int_{0}^{T_{N}} \int_{0}^{T_{N}}\left\langle N(t) N\left(t^{\prime}\right)\right\rangle d t d t^{\prime} \\
& =\frac{A_{f}^{2}}{T_{N}^{2}} \int_{0}^{T_{N}} \int_{0}^{T_{N}} \delta\left(t-t^{\prime}\right) d t d t^{\prime} \\
& =A_{f}^{2} / T_{N}
\end{aligned}
$$

The interpolated noise proposed in the main article [illustrated in Fig. 3(b)] can be written as

$$
N(t)=\sum_{i=1}^{\infty}\left[\left(1-\tau_{i}\right) X_{i}+\tau_{i} X_{i+1}\right] \Pi\left(\tau_{i}-1 / 2\right),
$$

where $X_{i}$ are normal distributed random numbers with zero mean, $\Pi$ is the hat function, and $\tau_{i}$ is defined as

$$
\tau_{i}=\frac{t-i \cdot T_{\mathrm{c}}}{T_{\mathrm{c}}} \in[0,1]
$$

The term $\bar{N}$ can be calculated by

$$
\begin{aligned}
\left.\bar{N}\right|_{0} ^{T_{N}} & =\frac{1}{T_{N}} \int_{0}^{T_{N}} N(t) d t \\
& =\frac{1}{T_{N}} \int_{0}^{T_{N}} \sum_{i=1}^{\infty}\left[\left(1-\tau_{i}\right) X_{i}+\tau_{i} X_{i+1}\right] \Pi\left(\tau_{i}-1 / 2\right) d t \\
& =\frac{T_{c}}{T_{N}} \int_{0}^{1}\left[\sum_{j=1}^{T_{N} / T_{\mathrm{c}}}\left(1-\tau_{i}\right) X_{j}+\tau_{i} X_{j+1}\right] d \tau_{i} \\
& =\left.\frac{T_{c}}{T_{N}} \sum_{j=1}^{T_{N} / T_{\mathrm{c}}}\left[\left(\tau_{i}-\frac{\tau_{i}^{2}}{2}\right) X_{j}+\frac{\tau_{i}^{2}}{2} X_{j+1}\right]\right|_{\tau_{i}=0} ^{\tau_{i}=1} \\
& =\frac{T_{c}}{2 T_{N}} \sum_{j=1}^{T_{N} / T_{\mathrm{c}}}\left(X_{j}+X_{j+1}\right) .
\end{aligned}
$$

When $T_{N} \gg T_{\mathrm{c}}$, this is approximately

$$
\left.\bar{N}\right|_{0} ^{T_{N}}=\frac{T_{c}}{T_{N}} \sum_{j=1}^{T_{N} / T_{\mathrm{c}}} X_{j}
$$

so the variance of $\bar{N}$ is then

$$
\operatorname{Var}(\bar{N})=\frac{T_{\mathrm{c}}}{T_{N}} \operatorname{Var}\left(X_{i}\right) .
$$


Because $\operatorname{Var}(\bar{N})$ should equal the theoretical result in Eq. (B1), we can obtain that

$$
\operatorname{Var}\left(X_{i}\right)=A_{f}^{2} / T_{\mathrm{c}} \text {. }
$$

A similar procedure can be applied to the spatial noise, and can be used to explain why we use $1 /\left(T_{\mathrm{c}} L_{\mathrm{c}}\right)$ to replace the $1 /(\triangle t \triangle z)$ in Sec. II B.

\section{APPENDIX C: F FOR THE MacCormack METHOD}

Two differential operators, $\triangle_{f}$ and $\Delta_{b}$, are introduced to represent forward and backward difference, respectively:

$$
\begin{aligned}
\triangle_{f} f & =\left(f_{i+1}-f_{i}\right) /\left(z_{i+1}-z_{i}\right), \\
\triangle_{b} f & =\left(f_{i}-f_{i-1}\right) /\left(z_{i}-z_{i-1}\right) .
\end{aligned}
$$

$\mathbf{F}$ is discretized by the forward difference for the predictor step, written as

$$
\mathbf{F}\left(u_{i}^{t}, h_{i}^{t}\right)=\left[\begin{array}{c}
-u_{i}^{t} \triangle_{f} u_{i}^{t}-\triangle_{f} p_{i}^{t}+\frac{3 \mathrm{Oh}}{\left(h_{i}^{t}\right)^{2}} \frac{\left(h_{i+1}^{t}\right)^{2} \triangle_{f} u_{i}^{t}-\left(h_{i}^{t}\right)^{2} \triangle_{b} u_{i}^{t}}{z_{i+1}-z_{i}}+\sqrt{\frac{6}{\pi}} \frac{\mathrm{Th} \sqrt{\mathrm{Oh}}}{\left(h_{i}^{t}\right)^{2}} \triangle_{f}\left(h_{i}^{t} N_{i}^{t}\right) \\
-u_{i}^{t} \triangle_{f} h_{i}^{t}-h_{i}^{t} \triangle_{f} u_{i}^{t} / 2
\end{array}\right],
$$

while the backward method is applied for $\overline{\mathbf{F}}$ :

$$
\begin{gathered}
\overline{\mathbf{F}}\left(\overline{u_{i}^{t+1}}, \overline{h_{i}^{t+1}}\right) \\
\quad=\left[\begin{array}{c}
-\overline{u_{i}^{t+1}} \triangle_{b} \overline{u_{i}^{t+1}}-\triangle_{b} \overline{p_{i}^{t+1}}+\frac{3 \mathrm{Oh}}{\left(\overline{h_{i}^{t+1}}\right)^{2}} \frac{\left(\overline{h_{i}^{t+1}}\right)^{2} \triangle_{f} \overline{u_{i}^{t+1}}-\left(\overline{h_{i-1}^{t+1}}\right)^{2} \triangle_{b} \overline{u_{i}^{t+1}}}{z_{i}-z_{i-1}}+\sqrt{\frac{6}{\pi}} \frac{\mathrm{Th} \sqrt{\mathrm{Oh}}}{\left(\overline{h_{i}^{t+1}}\right)^{2}} \triangle_{b}\left(\overline{h_{i}^{t+1}} N_{i}^{t}\right) \\
-\overline{u_{i}^{t+1}} \triangle_{b} \overline{h_{i}^{t+1}}-\overline{h_{i}^{t+1}} \triangle_{b} \overline{u_{i}^{t+1}} / 2
\end{array}\right] .
\end{gathered}
$$

\section{APPENDIX D: DERIVATION OF THE DIMENSIONLESS SLE-RP}

For the linear instability, we set $h=1+\hat{r}$ with $\hat{r} \ll 1$ to linerize the SLE [Eqs. (4) (6)],

$$
\frac{\partial^{2} \hat{r}}{\partial t^{2}}-3 \mathrm{Oh} \frac{\partial \hat{r}}{\partial t}+\frac{\hat{r}^{\prime \prime}+\hat{r}^{\prime \prime \prime \prime}}{2}=-\sqrt{\frac{3}{2 \pi}} \mathrm{Th} \sqrt{\mathrm{Oh}} \mathcal{N}^{\prime \prime} .
$$

Then a finite Fourier transform is applied to Eq. (D1) to get

$$
\frac{d^{2} R}{d t^{2}}+\alpha \frac{d R}{d t}+\beta R=\sqrt{\frac{3}{2 \pi}} \text { Th } \sqrt{\mathrm{Oh}} \cdot k^{2} N
$$

where

$$
\alpha=3 \mathrm{Oh} k^{2}, \quad \beta=\left(k^{4}-k^{2}\right) / 2,
$$

and the transformed variables are defined as follows:

$$
R(k, t)=\int_{0}^{L} \hat{r}(z, t) e^{-i k z} d z \quad \text { and } \quad N(k, t)=\int_{0}^{L} \mathcal{N}(z, t) e^{-i k z} d z
$$

The solution of Eq. (D2) is linearly decomposed into two parts:

$$
R=R_{\mathrm{LE}}+R_{\mathrm{fluc}} \text {. }
$$

The first part is the solution to the homogenous form of Eq. (D2) (i.e., with $\mathrm{Th}=0$ ) with some stationary initial disturbance (i.e., $R=R_{i}$ and $d R / d t=0$ at $t=0$ ). The solution to the 
homogeneous ODE is straightforward to obtain

$$
R_{\mathrm{LE}}=R_{i} e^{-a t / 2}[\cosh (c t / 2)+a \sinh (c t / 2) / c],
$$

where

$$
a=3 \mathrm{Oh} k^{2}, \quad c=\sqrt{\left(9 \mathrm{Oh}^{2}-2\right) k^{4}+2 k^{2}} .
$$

Equation (D4) is a solution to the standard lubrication equations (there is no fluctuating component) and is thus denoted $R_{\mathrm{LE}}$ in Eq. (D3). The second component of the solution arises from solving the full form of Eq. (D3) with zero initial disturbance; this part of the solution is solely due to fluctuations, and is thus denoted $R_{\text {fluc }}$. This is obtained by determining the homogeneous equation's impulse response,

$$
H(k, t)=2 \tau e^{-a t / 2} \sinh (c t / 2) / c,
$$

which due to the linear, time-invariant nature of the system, allows us to write

$$
R_{\text {fluc }}=\sqrt{\frac{3}{2 \pi}} \operatorname{Th} \sqrt{\mathrm{Oh}} k^{2} \int_{0}^{t} N(k, t-\mathcal{T}) H(k, \mathcal{T}) d \mathcal{T} .
$$

$R$ is both a random and complex variable with zero mean. Note, $R_{\mathrm{LE}}$ is also random as it develops from a random initial condition, but is uncorrelated with $R_{\text {fluc }}$ (both have zero mean). So the root mean square (rms) of $R$ is sought, which from Eq. (D3) is given by

$$
|R|_{\mathrm{rms}}=\sqrt{\overline{\left|R_{\mathrm{LE}}+R_{\mathrm{fluc}}\right|^{2}}}=\sqrt{\mid \overline{\left.R_{\mathrm{LE}}\right|^{2}}+\overline{\left|R_{\mathrm{fluc}}\right|^{2}}}
$$

where $\left|R_{\mathrm{LE}}\right|^{2}$ can easily be obtained from Eq. (D4),

$$
\overline{\left|R_{\mathrm{LE}}\right|^{2}}=\overline{\left|R_{i}\right|^{2}} e^{-a t}[\cosh (c t / 2)+a \sinh (c t / 2) / c]^{2} .
$$

Moreover, because $N$ is uncorrelated Gaussian white noise, and the variance of the norm of the white noise $\overline{|N|^{2}}=L$, Eqs. (D6) and (D7) combine to give

$$
\begin{aligned}
\overline{\left|R_{\text {fluc }}\right|^{2}} & =\left(\sqrt{\frac{3}{2 \pi}} \mathrm{Th} \sqrt{\mathrm{Oh}} k^{2}\right)^{2} \mid \overline{\left.\int_{0}^{t} N(k, t-\mathcal{T}) H(k, \mathcal{T}) d \mathcal{T}\right|^{2}}, \\
& =\left(\sqrt{\frac{3}{2 \pi}} \mathrm{Th} \sqrt{\mathrm{Oh}} k^{2}\right)^{2}\left(\int_{0}^{t} \overline{|N(k, t-\mathcal{T})|^{2}} H(k, \mathcal{T})^{2} d \mathcal{T}\right), \\
& =\left(\sqrt{\frac{3}{2 \pi}} \mathrm{Th} \sqrt{\mathrm{Oh}} k^{2}\right)^{2} L \int_{0}^{t} H^{2} d \mathcal{T}, \\
& =\frac{3 L}{\pi}\left(\mathrm{Th} \sqrt{\mathrm{Oh}} k^{2}\right)^{2} \frac{\left(a^{2}-c^{2}\right)-a^{2} \cosh (c t)-a c \sinh (c t)+c^{2} e^{a t}}{a c^{2}\left(a^{2}-c^{2}\right) e^{a t}} .
\end{aligned}
$$

Equations $(\mathrm{D} 8) \sim(\mathrm{D} 10)$ construct the SLE-RP model used in Sec. III A.

[1] O. A. Basaran, H. Gao, and P. P. Bhat, Nonstandard inkjets, Annu. Rev. Fluid Mech. 45, 85 (2013).

[2] S. Shabahang, J. Kaufman, D. Deng, and A. Abouraddy, Observation of the Plateau-Rayleigh capillary instability in multi-material optical fibers, Appl. Phys. Lett. 99, 161909 (2011).

[3] S. Mitragotri, Current status and future prospects of needle-free liquid jet injectors, Nat. Rev. Drug Discovery 5, 543 (2006).

[4] J. Eggers and E. Villermaux, Physics of liquid jets, Rep. Prog. Phys. 71, 036601 (2008). 
[5] J. A. F. Plateau, Statique expérimentale et théorique des liquides soumis aux seules forces moléculaires (Gauthier-Villars, Paris, 1873), Vol. 2.

[6] L. Rayleigh, On the instability of jets, Proc. London Math. Soc. 1, 4 (1878).

[7] Y. Li and J. E. Sprittles, Capillary breakup of a liquid bridge: Identifying regimes and transitions, J. Fluid Mech. 797, 29 (2016).

[8] J. B. Keller and M. J. Miksis, Surface tension-driven flows, SIAM J. Appl. Math. 43, 268 (1983).

[9] M. P. Brenner, J. Eggers, K. Joseph, S. R. Nagel, and X. Shi, Breakdown of scaling in droplet fission at high Reynolds number, Phys. Fluids 9, 1573 (1997).

[10] D. T. Papageorgiou, Analytical description of the breakup of liquid jets, J. Fluid Mech. 301, 109 (1995).

[11] D. T. Papageorgiou, On the breakup of viscous liquid threads, Phys. fluids 7, 1529 (1995).

[12] J. Eggers, Universal Pinching of 3D Axisymmetric Free-Surface Flow, Phys. Rev. Lett. 71, 3458 (1993).

[13] J. R. Castrejón-Pita, A. A. Castrejón-Pita, S. S. Thete, K. Sambath, I. M. Hutchings, J. Hinch, J. R. Lister, and O. A. Basaran, Plethora of transitions during breakup of liquid filaments, Proc. Natl. Acad. Sci. USA 112, 4582 (2015).

[14] A. Lagarde, C. Josserand, and S. Protière, Oscillating path between self-similarities in liquid pinch-off, Proc. Natl. Acad. Sci. USA 115, 12371 (2018).

[15] H. A. Stone, A. D. Stroock, and A. Ajdari, Engineering flows in small devices: Microfluidics toward a laboratory-on-a-chip, Annu. Rev. Fluid Mech. 36, 381 (2004).

[16] B. Derby, Inkjet printing of functional and structural materials: Fluid property requirements, feature stability, and resolution, Annu. Rev. Mater. Res. 40, 395 (2010).

[17] T. Gu and J. Hu, Chalcogenide glass metasurfaces from fluid instabilities, Nat. Nanotechnol. 14, 309 (2019).

[18] M. Moseler and U. Landman, Formation, stability, and breakup of nanojets, Science 289, 1165 (2000).

[19] Y. Hennequin, D. G. A. L. Aarts, J. H. van der Wiel, G. Wegdam, J. Eggers, H. N. W. Lekkerkerker, and D. Bonn, Drop Formation by Thermal Fluctuations at an Ultralow Surface Tension, Phys. Rev. Lett. 97, 244502 (2006).

[20] J. Petit, D. Rivière, H. Kellay, and J.-P. Delville, Break-up dynamics of fluctuating liquid threads, Proc. Natl. Acad. Sci. USA 109, 18327 (2012).

[21] Y. S. Choi, S. J. Kim, and M. U. Kim, Molecular dynamics of unstable motions and capillary instability in liquid nanojets, Phys. Rev. E 73, 016309 (2006).

[22] W. Kang and U. Landman, Universality Crossover of the Pinch-Off Shape Profiles of Collapsing Liquid Nanobridges in Vacuum and Gaseous Environments, Phys. Rev. Lett. 98, 064504 (2007).

[23] L. D. Landau, E. M. Lifshits, and L. P. Pitaevskii, in Statistical Physics, Part 2: Theory of The Condensed State, Course of Theoretical Physics, 2nd ed., Vol. 9 (Pergamon Press, Oxford, 1980), Chap. 9, pp. 86-91.

[24] C. Zhao, J. E. Sprittles, and D. A. Lockerby, Revisiting the Rayleigh-Plateau instability for the nanoscale, J. Fluid Mech. 861, R3 (2019).

[25] J. Eggers, Dynamics of Liquid Nanojets, Phys. Rev. Lett. 89, 084502 (2002).

[26] G. Grün, K. Mecke, and M. Rauscher, Thin-film flow influenced by thermal noise, J. Stat. Phys. 122, 1261 (2006).

[27] S. Nesic, R. Cuerno, E. Moro, and L. Kondic, Fully nonlinear dynamics of stochastic thin-film dewetting, Phys. Rev. E 92, 061002(R) (2015).

[28] J. A. Diez, A. G. González, and R. Fernández, Metallic-thin-film instability with spatially correlated thermal noise, Phys. Rev. E 93, 013120 (2016).

[29] M. A. Durán-Olivencia, R. S. Gvalani, S. Kalliadasis, and G. A. Pavliotis, Instability, rupture, and fluctuations in thin liquid films: Theory and computations, J. Stat. Phys. 174, 579 (2019).

[30] Y. Zhang, J. E. Sprittles, and D. A. Lockerby, Molecular simulation of thin liquid films: Thermal fluctuations and instability, Phys. Rev. E 100, 023108 (2019).

[31] M. Gallo, F. Magaletti, and C. M. Casciola, Thermally activated vapor bubble nucleation: The LandauLifshitz-Van der Waals approach, Phys. Rev. Fluids 3, 053604 (2018).

[32] S. Plimpton, Fast parallel algorithms for short-range molecular dynamics, J. Comput. Phys. 117, 1 (1995).

[33] M. S. Green, Markoff random processes and the statistical mechanics of time-dependent phenomena. II. Irreversible processes in fluids, J. Chem. Phys. 22, 398 (1954). 
[34] R. Kubo, Statistical-mechanical theory of irreversible processes. I. General theory and simple applications to magnetic and conduction problems, J. Phys. Soc. Japan 12, 570 (1957).

[35] J. G. Kirkwood and F. P. Buff, The statistical mechanical theory of surface tension, J. Chem. Phys. 17, 338 (1949).

[36] A. Trokhymchuk and J. Alejandre, Computer simulations of liquid/vapor interface in lennard-jones fluids: Some questions and answers, J. Chem. Phys. 111, 8510 (1999).

[37] A. Ghoufi, P. Malfreyt, and D. J. Tildesley, Computer modeling of the surface tension of the gas-liquid and liquid-liquid interface, Chem. Soc. Rev. 45, 1387 (2016).

[38] L. Korson, W. Drost-Hansen, and F. J. Millero, Viscosity of water at various temperatures, J. Chem. Phys. 73, 34 (1969).

[39] R. T. Cygan and J. D. Kubicki, Molecular Modeling Theory: Applications in the Geosciences (Walter de Gruyter, Berlin, 2018), Vol. 42.

[40] V. Molinero and E. B. Moore, Water modeled as an intermediate element between carbon and silicon, J. Chem. Phys. 113, 4008 (2008).

[41] J. L. Abascal and C. Vega, A general purpose model for the condensed phases of water: Tip4p/2005, J. Chem. Phys. 123, 234505 (2005).

[42] J. Eggers and T. F. Dupont, Drop formation in a one-dimensional approximation of the Navier-Stokes equation, J. Fluid Mech. 262, 205 (1994).

[43] A. Donev, E. Vanden-Eijnden, A. Garcia, and J. Bell, On the accuracy of finite-volume schemes for fluctuating hydrodynamics, Commun. Appl. Math. Comput. Sci. 5, 149 (2010).

[44] J. B. Bell, A. L. Garcia, and S. A. Williams, Numerical methods for the stochastic Landau-Lifshitz NavierStokes equations, Phys. Rev. E 76, 016708 (2007).

[45] R. MacCormack, The effect of viscosity in hypervelocity impact cratering, J. Spacecraft Rockets 40, 757 (2003).

[46] D. G. Aarts, M. Schmidt, and H. N. Lekkerkerker, Direct visual observation of thermal capillary waves, Science 304, 847 (2004).

[47] A. Willis and J. Freund, Thermal capillary waves relaxing on atomically thin liquid films, Phys. Fluids 22, 022002 (2010).

[48] X. Xue, M. Sbragaglia, L. Biferale, and F. Toschi, Effects of thermal fluctuations in the fragmentation of a nanoligament, Phys. Rev. E 98, 012802 (2018).

[49] N. Gopan and S. P. Sathian, Rayleigh instability at small length scales, Phys. Rev. E 90, 033001 (2014).

[50] D. K. Bhuptani and S. P. Sathian, Effect of axial electric field on the Rayleigh instability at small length scales, Phys. Rev. E 95, 053115 (2017).

[51] D. Rutland and G. Jameson, A nonlinear effect in the capillary instability of liquid jets, J. Fluid Mech. 46, 267 (1971).

[52] J. Becker, G. Grün, R. Seemann, H. Mantz, K. Jacobs, K. R. Mecke, and R. Blossey, Complex dewetting scenarios captured by thin-film models, Nat. Mater. 2, 59 (2003).

[53] R. Fetzer, M. Rauscher, R. Seemann, K. Jacobs, and K. Mecke, Thermal Noise Influences Fluid Flow in Thin Films During Spinodal Dewetting, Phys. Rev. Lett. 99, 114503 (2007). 